\title{
Ideal Negative Measurements in Quantum Walks Disprove Theories Based on Classical Trajectories
}

\author{
Carsten Robens, ${ }^{1}$ Wolfgang Alt, ${ }^{1}$ Dieter Meschede, ${ }^{1}$ Clive Emary, ${ }^{2}$ and Andrea Alberti ${ }^{1, *}$ \\ ${ }^{1}$ Institut für Angewandte Physik, Universität Bonn, Wegelerstr. 8, D-53115 Bonn, Germany \\ ${ }^{2}$ Department of Physics and Mathematics, University of Hull, \\ Kingston-upon-Hull HU6 7RX, United Kingdom
}

(Received 15 June 2014; revised manuscript received 13 November 2014; published 20 January 2015)

\begin{abstract}
We report on a stringent test of the nonclassicality of the motion of a massive quantum particle, which propagates on a discrete lattice. Measuring temporal correlations of the position of single atoms performing a quantum walk, we observe a $6 \sigma$ violation of the Leggett-Garg inequality. Our results rigorously excludes (i.e., falsifies) any explanation of quantum transport based on classical, well-defined trajectories. We use so-called ideal negative measurements - an essential requisite for any genuine Leggett-Garg test- to acquire information about the atom's position, yet avoiding any direct interaction with it. The interactionfree measurement is based on a novel atom transport system, which allows us to directly probe the absence rather than the presence of atoms at a chosen lattice site. Beyond the fundamental aspect of this test, we demonstrate the application of the Leggett-Garg correlation function as a witness of quantum superposition. Here, we employ the witness to discriminate different types of walks spanning from merely classical to wholly quantum dynamics.
\end{abstract}

DOI: 10.1103/PhysRevX.5.011003

\section{INTRODUCTION}

The superposition principle is one of the pillars of quantum theory, and it also constitutes a central resource in quantum metrology [1], quantum communication technologies [2], and quantum information processing [3]. Yet the same principle has been the source of heated discussions since the inception of quantum theory [4-14]: The central question of the long-standing debate is about the physical origin of the observed "definiteness" of macroscopic physical objects. In fact, while it is widely accepted that microscopic systems can live in superposition states, the fact that in a physical apparatus individual measurements always yield single, definite outcomes has so far eluded a comprehensive explanation [15]. To reconcile the definiteness of measurements with the Schrödinger equation, two plausible explanations have been advanced [16]: (1) Quantum superposition applies at all scales, even for macroscopic objects, and environment-induced decoherence is responsible for the emergence of so-called pointer states, to which the wave function is reduced ("collapses") with probabilities determined by Born's rule.

(2) There exists a deeper, underlying theory that gives rise

\footnotetext{
*alberti@iap.uni-bonn.de; http://quantum-technologies.iap .uni-bonn.de.

Published by the American Physical Society under the terms of the Creative Commons Attribution 3.0 License. Further distribution of this work must maintain attribution to the author(s) and the published article's title, journal citation, and DOI.
}

Subject Areas: Quantum Physics

to coherent quantum evolution at the microscale and yet well-defined trajectories at the macroscopic level, independently of the environment's influence. This second explanation advocates a "macrorealistic" description of nature as it implies that macroscopic physical objects follow classical trajectories.

In order to put the latter idea of macrorealism to the experimental test, Leggett and Garg (LG) derived a set of inequalities bounding the linear combinations of two-time correlation measurements [17]. In recent years, violation of LG inequalities has been shown in a wide range of physical systems spanning from superconducting qubits $[18,19]$ to photons [20-23], nitrogen-vacancy centers in diamond [24], nuclear spins [25], and phosphorus impurities in silicon [26]. However, these experiments are confined to test superposition states in a simple qubit system, which exhibits Rabi oscillations-far away from Leggett and Garg's original intention to probe macroscopic quantum superpositions.

Performing LG tests in more complex systems including also mechanical degrees of freedom-mechanical superposition states are the essential component of most macrorealistic models [27-29] — constitutes a major challenge: Not only do quantum superposition states become very fragile, but new experimental methods must also be developed to realize so-called "ideal negative measurements" in these systems. Ideal negative measurementsnamely, the ability to measure the physical object yet avoid any direct interaction with it - are a prerequisite for any rigorous LG test, as without it, violations can simply be 
attributed to an unwitting invasiveness on behalf of the experimenter, rather than to the absence of a realistic description [30]. Despite their importance, a rigorous implementation of this type of measurement has been demonstrated in just one of the many LG tests reported in the literature [26].

In this article, we report on a $6 \sigma$ (standard deviation) violation of LG inequality for a cesium atom performing a so-called "quantum walk," in which the atom is coherently transported along a line in discrete steps in space and time. We obtain the violation by measuring the correlation between the atom's positions at successive times with measurements of the ideal negative type, which a devout realist would perceive as noninvasive. Our protocol for ideal negative measurements rests upon a novel atom transport technology consisting of two optical lattice potentials that are fully independent, though perfectly stabilized to each other. The capability of the new system to state-dependently displace atoms over arbitrary large distances enables us to remove atoms depending on their position state and to realize, thus, a negative position measurement on the unshifted atoms.

Criteria for the assessment of the degree of macroscopicity of superposition states have long been discussed in the literature [31,32]. There is a general agreement that the macroscopicity of a mechanical system increases with heavier masses and larger spatial separations of the superposition states. Although the atomic wave function of the cesium atom in our experiment spreads, at most, over a distance of 5 sites $(2 \mu \mathrm{m})$, our results set the stage for future experiments testing the LG inequalities with objects of thousands of proton masses split over macroscopic distances (for a review, see Ref. [33]). Furthermore, we remark that this work extends the experimental study of LG violations to quantum transport systems [34] with dynamics far richer than those of the hitherto-considered qubit systems.

\section{QUANTUM TRANSPORT}

Introduced by Richard Feynman to model the one-dimensional motion of a spin-1/2 particle [35], discrete-time quantum walks can be regarded as the archetype of quantum transport experiments. While quantum walks share many similarities with classical random walks, the behavior of these two transport paradigms is strongly different.

In a "classical" random walk scenario, a particle moves in discrete steps, either leftward or rightward, with the direction determined by the result of a coin toss. After iterating the sequence of coin toss and subsequent displacement $n$ times, one finds the binomial distribution $\left(\begin{array}{l}n \\ x\end{array}\right) / 2^{n}$ describing the motion of the particle by simply enumerating the trajectories terminating in position $x$. The Brownian motion of colloidal particles suspended in a liquid is a well-known example of this type of diffusive classical transport.
A different scenario-which we call "quantum" in the light of the anticipated violation of the LG inequality-is instead realized by a cesium atom, which undergoes quantum diffusion in a one-dimensional optical lattice potential. Rather than tossing a real coin, a microwave "coin" pulse $\mathcal{C}$ is used to put the particle into an equal superposition of two internal hyperfine states of the electronic ground state, $\left|F=4, m_{F}=4\right\rangle$ and $\mid F=3$, $\left.m_{F}=3\right\rangle$, which we label for the sake of convenience as $\uparrow$ and $\downarrow$, respectively. While a quantum physicists would describe $\mathcal{C}$ as a $\pi / 2$ rotation of a pseudo spin- $1 / 2$ system, a devout realist would interpret $\mathcal{C}$ as a stochastic process that prepares the atom in one of the two internal states with equal probability-just like the coin toss. A statedependent shift operation $\mathcal{S}$ subsequently moves the atom by one site rightward or leftward depending on the internal state. As a result of this operation, an atom which is in the $\uparrow$ state moves from $x$ to $x-1$, while an atom in the $\downarrow$ state moves to $x+1$ instead.

The different sensitivity (ac polarizability) of the $\uparrow$ and $\downarrow$ states to left- and right-handed polarized light can be exploited for controlling the atom's position with statedependent optical potentials, each of which acts on either one of the two internal states (see also Appendix A) [36]. As illustrated in Fig. 1, this idea permits us to realize the shift $\mathcal{S}$ by means of two state-dependent optical lattices, whose position is independently controlled with subnanometer precision. Hence, the alternation of $\mathcal{C}$ and $\mathcal{S}$

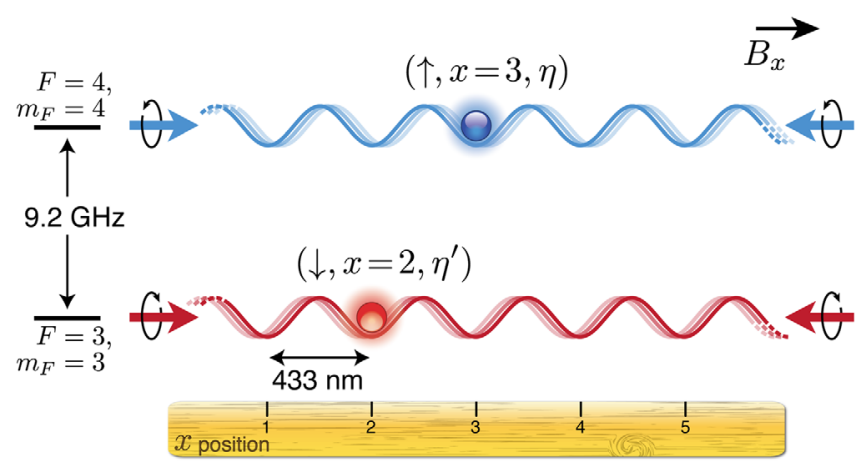

FIG. 1. Transport of single Cs atoms in state-dependent periodic potentials. Two independent optical lattices originate from standing waves of opposite circular polarization but identical wavelength $\lambda=866 \mathrm{~nm}$. Depending on the internal state, $\uparrow$ or $\downarrow$, atoms experience one or the other lattice potential. An optoelectronic servo-lock loop allows the position of each lattice to be arbitrarily controlled. The atom's position is retrieved with single-site resolution by fluorescence imaging. The parameter $\eta$ accounts for other degrees of freedom, such as the atom's position perpendicular to the lattice or, in general, other hidden physical aspects. The quantization axis is defined by the small bias magnetic field $B_{x}$, which is chosen along the two optical lattices. $F$ and $m_{F}$ denote, respectively, the total angular momentum and its projection along the quantization axis for both internal hyperfine states. 
operations realizes a one-dimensional discrete-time quantum walk.

As revealed in our first implementation of quantum walks [37], as well as in several other implementations using diverse physical systems [38-42], the spatial probability distribution of the quantum walk expands linearly with the number of steps $n$, in stark contrast to the $\sqrt{n}$ behavior of the classical random walk. Furthermore, prominent peaks are visible on either one or both sides of the distribution, depending on the initial internal state.

Quantum mechanics gives a precise account of these phenomena in terms of interference of all trajectories that the particle is allowed to follow while moving from the initial to the final point. The agreement with experimental observations, in the spirit of Francis Bacon's inductive thinking, serves as an important piece of validation of quantum theory itself. However, according to Karl Popper's point of view, one must acknowledge that the remarkable fit between observations and quantum theory does not itself constitute a "falsification" of the "other" hypothesis - that an underlying probability distribution could conceivably describe, at all times, the position and the spin of the atom as elements of objective reality.

\section{LEGGETT-GARG INEQUALITY}

Here is where the LG inequality becomes important, as it subjects the idea of realism to a rigorous, objective test by looking for violation of

$$
K=\left\langle Q\left(t_{2}\right) Q\left(t_{1}\right)\right\rangle+\left\langle Q\left(t_{3}\right) Q\left(t_{2}\right)\right\rangle-\left\langle Q\left(t_{3}\right) Q\left(t_{1}\right)\right\rangle \leq 1,
$$

where $Q\left(t_{i}\right)$ are real values with $\left|Q\left(t_{i}\right)\right| \leq 1$ assigned to the outcomes of a measurement performed at time $t_{i}$ with $t_{i}<t_{i+1}$, and where $\langle\ldots\rangle$ denotes the average over many repetitions of the experiment. The derivation of this inequality essentially rests on two assumptions [43]: (A1) realism, as above; and (A2) noninvasive measurability, which asserts the possibility to measure the system without affecting its future evolution. Both these assumptions are implicit in a realistic view of nature [17]; but of course, quantum mechanics does not hold to either [44,45]. However, to be a valid test of the LG inequality, it is sufficient to persuade those who already believes in (A1) that the measurement scheme used in the experiment complies with (A2). Otherwise, violations of Eq. (1) may be attributed to a trivial invasivity of the measurement [30]. To ensure this, Leggett and Garg put forward the concept of "ideal negative measurements" [17], which are well illustrated by the following example: Imagine that a physical object, like the atom, can be found in only two positions, $x= \pm 1$, and that we check the presence of the object at $x=+1$ without looking at $x=-1$. From the point of view of a realist, the absence of the object at $x=+1$ necessarily implies that $x=-1$ without ever having influenced the object during the measurement. By repeating this measurement many times, probing the object either at $x=+1$ or $x=-1$ and discarding all measurements that directly reveal the object, we can thus measure correlation functions like $\left\langle Q\left(t_{3}\right) Q\left(t_{2}\right)\right\rangle$ without having ever meddled with the object itself at time $t_{2}$. Hence, any violation of Eq. (1) that arises from ideal negative measurements must imply a violation of the realist principles (A1) or (A2)—or both.

\section{QUANTUM WALKS FALSIFY CLASSICAL TRAJECTORIES}

We base our experiment on a four-step quantum walk probed at times $t_{1}=0, t_{2}=1$, and $t_{3}=4$ steps, as displayed in the panels of Fig. 2, where each step lasts around $26 \mu \mathrm{s}$. The three different measurements are defined as follows. We equate the first measurement $Q\left(t_{1}\right)$ with the state preparation in $(\uparrow, x=0)$ : Fluorescence imaging first determines the initial position of the atom with single-site resolution [46], while sideband cooling slows the atom's motion to the lowest longitudinal vibrational state and concurrently polarizes the atom in the $\uparrow$ state [47]. The translational symmetry of the optical standing wave allows us to safely label the initial position with $x=0$. We designate $Q\left(t_{1}\right)=1$. At time $t_{2}$, we measure the atom's state, which is restricted to two possibilities, either $(\uparrow, x=-1)$ or $(\downarrow, x=+1)$, and we assign to this measurement the value $Q\left(t_{2}\right)=1$ independently of the atom's internal state or position. The assignment of $Q\left(t_{2}\right)$ to a constant value is, in fact, one of the legitimate choices that are consistent with the condition $\left|Q\left(t_{i}\right)\right| \leq 1$ in the derivation of LG inequalities [43]. Finally, $Q\left(t_{3}\right)$ measures the atom's position at the end of the walk and returns the value -1 for $x \leq 0$ and the value +1 for $x>0$. According to quantum mechanics, with this definition of $Q\left(t_{i}\right)$, we expect a violation of the LG inequality yielding $K=1.5$ (see Appendix F).

Quantum mechanics also shows that other designations of $Q\left(t_{2}\right)$ are possible to produce a violation of Eq. (1), for instance, by assigning the measurement outcome $(\downarrow, x=+1)$ to 1 and $(\uparrow, x=-1)$ to a certain value $\xi$ with $|\xi| \leq 1$. While previous experiments [18-26] have adopted dichotomic designations of $Q\left(t_{2}\right)$ (analogous to setting $\xi=-1$ here), we have intentionally dropped such an extra condition to permit larger violations of the LG inequality as fewer constraints are imposed [cf. Eqs. (F1) and (F2) in Appendix F]. Such a constant designation especially reveals that the essential requisite to violate Eq. (1) is that the particle is measured at $t_{2}$, even though the result of the measurement itself is then discarded.

Because the measurement $Q\left(t_{1}\right)$ is a state preparation, and because we are not concerned about the atom's evolution after time $t_{3}$, only the measurement $Q\left(t_{2}\right)$ must be performed noninvasively. Since we are not allowed to directly image the atom at time $t_{2}$ because it would be 

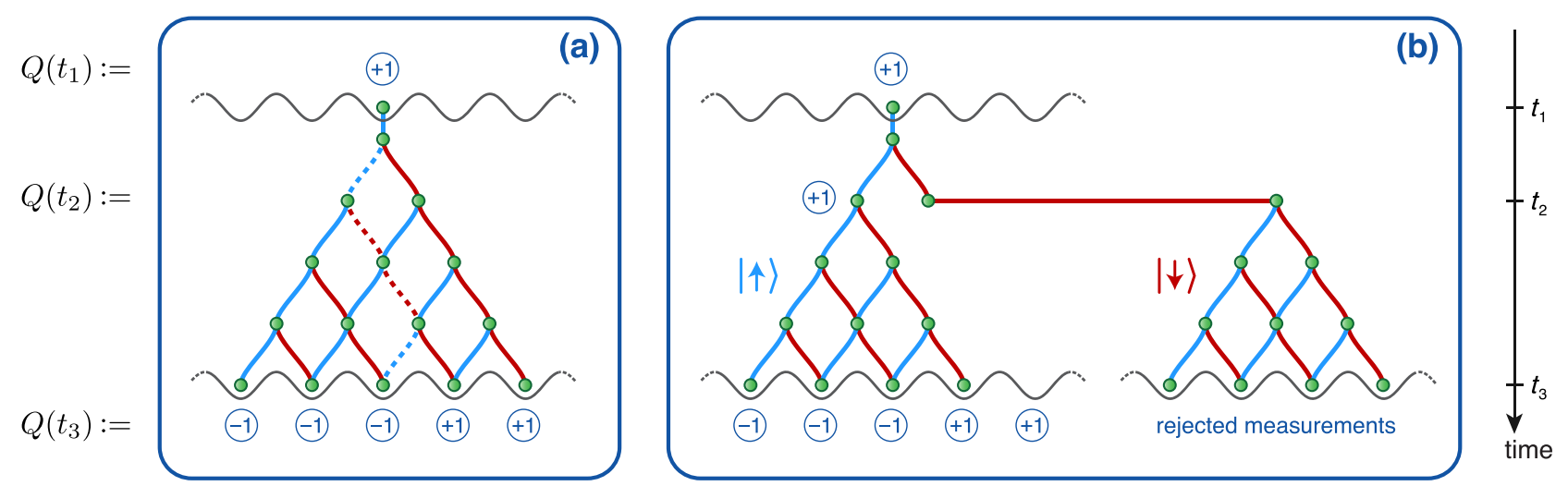

FIG. 2. Ideal negative measurements test the nonclassicality of quantum walks. (a) Schematic representation of a four-step quantum walk containing 16 possible trajectories, which according to quantum mechanics, the Cs atom simultaneously follows. Conversely, upholders of realism believe that in each experiment the atom follows a definite trajectory connecting the initial and final point, e.g., the dashed line shown in the figure. The outcomes \pm 1 of $Q\left(t_{i}\right)$ measurements are indicated with circles, where $Q\left(t_{1}\right)$ is identified with the initial-state preparation, whereas $Q\left(t_{2}\right)$ and $Q\left(t_{3}\right)$ are related to position measurements. For instance, measurements at times $t_{1}$ and $t_{3}$ yield the correlation function $\left\langle Q\left(t_{3}\right) Q\left(t_{1}\right)\right\rangle$. (b) To measure the correlation function $\left\langle Q\left(t_{3}\right) Q\left(t_{2}\right)\right\rangle$, we use at time $t_{2}$ an ideal negative measurement scheme, which ensures the noninvasiveness of $Q\left(t_{2}\right)$ : On the condition that only atoms in the $\downarrow$ state are transported at $t_{2}$ far away to the right, atoms in $\uparrow$ continue their walk undisturbed. In case $Q\left(t_{2}\right)$ measurement has not removed the atom, measuring at $t_{3}$ the atom's position yields $Q\left(t_{3}\right)$ conditioned to the state $(\uparrow, x=-1)$ at $t_{2}$. Likewise, we obtain $Q\left(t_{3}\right)$ conditioned to $(\downarrow, x=+1)$ by transporting at $t_{2}$ the atom in $\downarrow$ far away to the left (not shown in the figure).

invasive, we adopt an ideal negative measurement strategy that hinges on state-selective removal of atoms. This measurement scheme draws direct inspiration from the experimental realization of interaction-free measurements of the state of single photons [48]. The measurement scheme, which is illustrated in Fig. 2(b), proceeds as follows: If we want to noninvasively detect the atom's presence, say, in $x=-1$, we remove the atoms in the state $(\downarrow, x=+1)$ by transporting them far to the right, whereas we leave the atoms in the state $(\uparrow, x=-1)$ untouched. Provided that this shift (set here to 5 sites) is larger than the distance covered by the atom between $t_{2}$ and $t_{3}$, the atom's position at the later time $t_{3}$ allows us to unequivocally mark the shifted atoms (which remain trapped in the lattice potential) as effectively removed with confidence better than $99 \%$. Hence, the state-selective removal of atoms provides information about the atom's position at time $t_{2}$ and, at the same time, postselects those measurements that are carried out noninvasively.

In the experiment, state-selective removal of atoms requires the ability to shift one single spin species at a time over arbitrary distances. However, previous implementations of state-dependent transport have so far only demonstrated the concurrent shift of both spin species instead of an individual one [36,37,49]. Moreover, the largest displacement attained heretofore with a single transport operation amounts to about one lattice site [36]. We overcome these limitations by employing a new atom transport technology, which relies on two spatially overlapped, yet fully independent optical lattices. In the new implementation, the two optical standing waves that create the lattice potentials (see also Fig. 1) originate from independent laser beams with opposite circular polarizations, whose phase and frequency can individually be controlled with the aid of acousto-optic modulators. Two optical phase-lock loops are employed to stabilize the position of both periodic lattices against a common third reference laser beam. We thereby achieve a stability of the relative position between the two lattices on the level of $100 \mathrm{pm}$ to be compared with the $20-\mathrm{nm}$ localization of the atoms along the lattice direction. The complete independence of the two standing waves allows us to arbitrarily control the position of each lattice by varying the phase of the corresponding laser beams. The intensity of each laser beam is actively stabilized to better than $0.1 \% \mathrm{rms}$ noise.

In order to measure the LG correlation function, we note that with our assignment of $Q\left(t_{i}\right)$, the correlation function $K_{12} \equiv\left\langle Q\left(t_{2}\right) Q\left(t_{1}\right)\right\rangle$ is trivially equal to 1 . Furthermore, we have $K_{13} \equiv\left\langle Q\left(t_{3}\right) Q\left(t_{1}\right)\right\rangle=\left\langle Q\left(t_{3}\right)\right\rangle$, which quantifies the asymmetry of the final position distribution. Figure 3(a) shows the measured probability distribution of a four-step quantum walk with fair coin toss $(\theta=\pi / 2)$. The distribution is characterized by a pronounced skew to the left, which translates into a nonzero value $K_{13}=-0.57 \pm 0.05$. Although this asymmetry itself is often interpreted as a hallmark of "quantumness" [37,38], we would rather eschew similar premature conclusions here. Using the law of total probability under assumptions (A1) and (A2), the final correlation function may be obtained as

$$
K_{23}=\sum_{x= \pm 1} P\left(t_{2} ; x\right)\left\langle Q\left(t_{3}\right)\right\rangle_{x}
$$



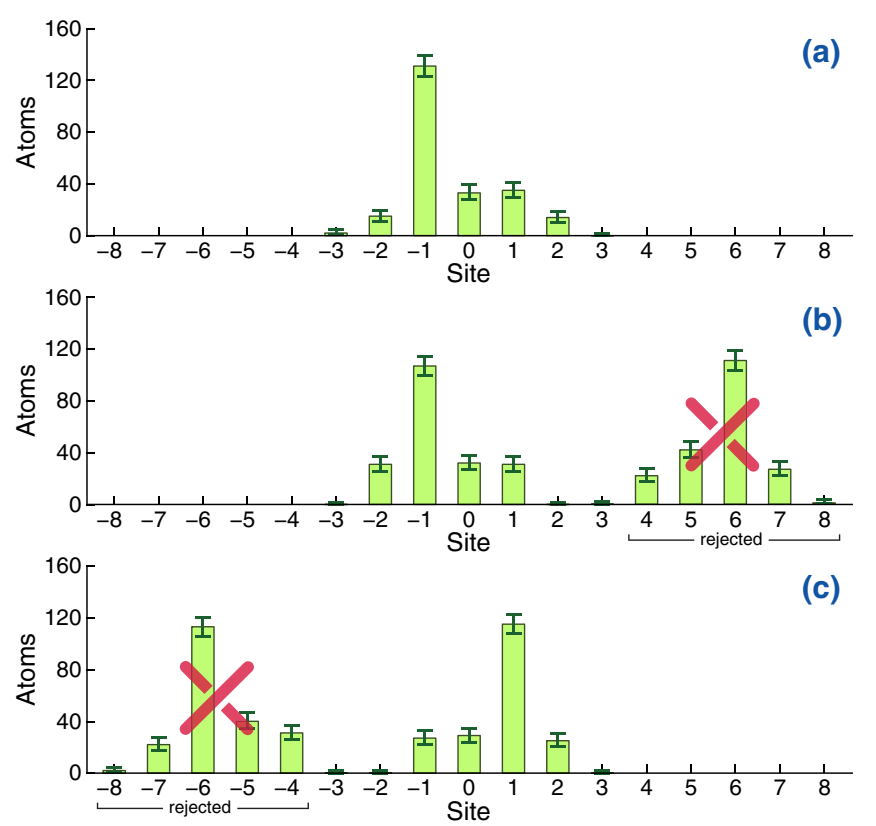

FIG. 3. Violation of Leggett-Garg inequality probing a fourstep quantum walk. The spatial distribution of single atoms is reconstructed by measuring their positions at time $t_{3}$ : (a) If we do not observe which trajectory the atom has taken at $t_{2}$, the distribution exhibits a pronounced peak on the left-hand side. However, when we conclude from an ideal negative result whether the atom at time $t_{2}$ was in (b) $x=-1$ or (c) $x=1$, we obtain two distributions that resemble the mirror image of one other. The events in which the atom's position has been affected by $Q\left(t_{2}\right)$ measurement are recognized through the larger displacement and, thus, rejected. Because the overall number of probed atoms, 404, is the same in (b) and (c), the retained events can be added together to produce the position distribution at $t_{3}$ conditioned on having measured the position at $t_{2}$. The sum distribution (not shown) is symmetric and differs strongly from the asymmetric distribution in (a). The vertical error bars represent $68 \%$ Clopper-Pearson confidence intervals.

where $P\left(t_{2} ; x\right)$ is the probability of finding the atom in $x$ at $t_{2}$, and $\langle\ldots\rangle_{x}$ is the average over the distribution conditioned on a negative detection of the atom in $x$ at $t_{2}$. Hence, we perform two separate experiments to measure $K_{23}$, one for each term of the sum in Eq. (2), as shown in Fig. 3(c). After rejecting all measurements during which atoms have provably been perturbed, we find $P\left(t_{2} ; x=-1\right)=0.506 \pm$ 0.026 and $P\left(t_{2} ; x=+1\right)=0.494 \pm 0.026$. Averaging $Q\left(t_{3}\right)$ with the two conditioned distributions yields a value $K_{23}=-0.14 \pm 0.05$ close to zero. Taken together, the three correlation functions yield $K=1.435 \pm 0.074>1$, which violates the LG inequality by about $6 \sigma$. The uncertainty is estimated to be purely statistical (see Appendix C).

\section{QUANTUM WITNESS}

Besides the fundamental interest, LG inequalities also find application in quantifying the degree of quantumness of a system. This requires, however, that we abandon the standpoint of realists and, from now on, embrace quantum mechanics instead. Intuitively, the LG correlation function $K$ may serve as an indicator, say a witness, of the amount of superposition involved in the system's dynamics. This idea of "quantum witnesses" has recently been proposed as a method to discern quantum signatures in systems like biological organisms [50].

Owing to our particular definition of $Q\left(t_{2}\right)$, which is constantly mapped to 1 , we prove a direct connection (see Appendix G) between LG inequalities and quantum witness formalism by identifying $W \equiv|K-1|$ with the first quantum witness introduced in Ref. [50]. The deviation of $W$ from zero indicates the degree of quantumness in the system's dynamics.

We provide demonstration of the quantum witness $W$ in the four-step quantum walk by testing different types of coins, which differ in the probability of tails $p=\cos ^{2}(\theta / 2)$ and heads $q=1-p$. For instance, $p=q=1 / 2$ corresponds to the fair coin's situation, which has hitherto been considered. As displayed in Fig. 4, we measure the LG correlation function $K$ for different values of the coin angle $\theta$, which is tuned by setting the duration of the coin's microwave pulse. The violation is maximal for $\theta=\pi / 2$ (fair coin), when the coin maximally splits the walker's state at each step in an equal superposition of states. Instead, the violation vanishes for $\theta=0$ and $\theta=\pi$, when the walk reduces to classic transport with no superposition involved.

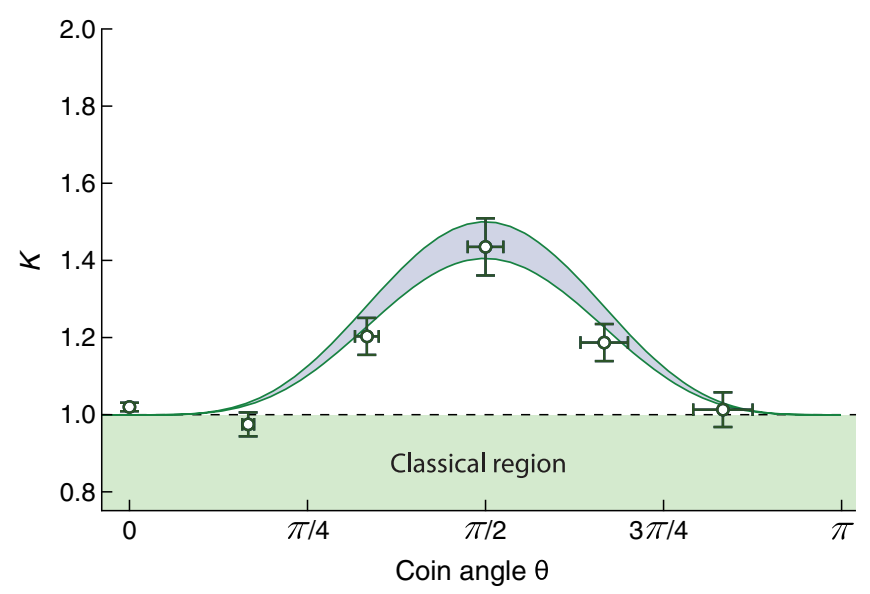

FIG. 4. Leggett-Garg correlation measurement witnessing the degree of quantumness. Maximum violation occurs for a fair coin $(\theta=\pi / 2)$, while no violation occurs for classical transport at $\theta=0$ and $\theta=\pi$. The solid line is the theoretical prediction based on quantum mechanics of the LG correlation function $K$ for a decoherence-free quantum walk (upper curve) and for a quantum walk with $10 \%$ decoherence per step (lower curve). The vertical error bars represent $1 \sigma$ uncertainty, while the horizontal error bars denote a systematic uncertainty on the coin angle. 


\section{INTERPRETATION AND DISCUSSION}

The reported violation of the LG inequality proves that the concept of a well-defined, classical trajectory is incompatible with the results obtained in a quantum-walk experiment. Yet, the concept of well-defined trajectories in position space can, in part, still be rescued, provided that one renounces locality. An example is provided by Bohmian mechanics, whose predictions are shown to be equivalent to those of nonrelativistic quantum mechanics [51]. In this interpretation of quantum theory, physical objects follow precise trajectories, which are guided by the Universe's pilot wave function, that is, by a physical entity constituting a nonlocal hidden variable. It is therefore clear that Bohmian mechanics is not in contradiction with our findings since, from that point of view, assumption (A2) is not fulfilled.

Recently, a minimal macrorealistic extension of (nonrelativistic) quantum mechanics has been put forward under general assumptions [32], proposing a universal objective measure of macroscopicity accounting for both the mass and spatial separation of the superposition states. Within this model, we estimate a measure of macroscopicity for our experiment (see Appendix H) that lies in the range of typical cold-atom experiments [33] — whether they are performed with thermal atoms or with a Bose-Einstein condensate. We remark, moreover, that the macroscopicity of our experiment is, coincidentally, on the same order of magnitude of experiments testing superpositions of macroscopic persisting currents $[18,33,52]$. In spite of the yet microscopic nature of the present LG tests, our result gives a conceptual demonstration that noninvasive measurement techniques can be applied to test the LG inequality, e.g., in double-slit experiments with genuinely massive particles by alternatively blocking at time $t_{2}$ either one of the two slits.

Unlike the test of Bell inequalities, where a loophole-free violation seems in reach [53], LG experiments remain susceptible to the so-called clumsiness loophole-even employing negative measurements. This loophole refers to the impossibility on behalf of the experimenter to exclude an invasivity of the measurements. Hence, it is appropriate to comment on the three main instances that can hinder the fulfillment of (A2) in our experimental setup. (1) In the measurement of $Q\left(t_{2}\right)$, the state-dependent shift could cause motional excitations to the unshifted atoms. To avoid this problem, we deliberately set the shift duration to a time of $200 \mu \mathrm{s}$, which is much longer than the period of the longitudinal motion of $\approx 10 \mu \mathrm{s}$. We measured the fraction of atoms that are left in the ground state by the shift process for both shifted and unshifted internal states [47]. In both cases, we obtained a fraction $>99 \%$, which is consistent with the precision of the initial preparation, thus confirming that no excitation is produced. The concept of venality, which has been introduced in Ref. [26] to account for nonideal negative measurements, can be applied to this effect as well. The analysis in Appendix E, however, shows that the upper limit imposed on $K$ is only slightly changed. (2) The duration of measurement $Q\left(t_{2}\right)$ is comparable to the spin coherence time. In principle, an equal delay time should also be included in the sequence when no measurement is performed at $t_{2}$. Even doing so, we verified using a different experimental sequence, a Ramsey interferometer instead of a quantum walk, that a violation of the LG inequality is still produced. (3) At time $t_{1}$, the motion of the atom in the transverse direction is prepared according to a Boltzmann-like distribution, which extends over the first hundred motional states. A statistical mixture is not a problem per se, provided that the statistical properties are maintained constant. A realist, though, could raise the objection that the experiment "knows" which correlation term, either $K_{13}$ or $K_{23}$, is being measured and exploits this information to prepare the transverse motion ad hoc in a way to counterfeit the violation of the LG inequality (cf. the hypothesis of so-called induction discussed by Leggett in Ref. [15]). More generally, the same argument can also be invoked in the case of any hidden variable $\eta$, which, from an epistemological point of view, is tantamount to the transverse motion of the atoms. Eventually, to blunt this criticism, one could base the choice of which correlation term to measure upon random events that are uncorrelated from the initial preparation $[54,55]$.

There is one further aspect of this LG test that must be emphasized, namely, that we test single, individual copies of the system by probing one cesium atom at a time. Prior experiments in NMR systems $[25,26]$ took an alternative approach by substituting individual measurements with measurements on a large ensemble of identical systems instead. Our approach a priori eliminates the need for the extra assumption that multiple copies of the system-even when positioned in near proximity-do not interact with each other. However plausible this hypothesis is in NMR systems, ignoring it would allow a realist to argue that the several copies of the system have interacted with each other-in particular, with those copies that have been invasively measured, thus invalidating hypothesis (A2). In addition, employing ensembles instead of individual systems can lead to controversial interpretations, as is illustrated by the following examples. A wavelike analogue of quantum walks based on coherent electromagnetic waves (e.g., a laser beam [41]) is expected to produce a violation of the LG inequality similar to the one obtained with individual photons. In a similar way, even acoustic or surface waves could be used to measure a violation. However, it is certainly debatable whether an experiment hinging on Maxwell equations or mechanical waves can indeed rule out realism. In fact, to reach this conclusion, a realist should first be persuaded that light is composed of photons and waves of phonons.

In conclusion, our experiment gives a rigorous, quantitative demonstration of the nonclassicality of a 
massive-particle quantum walk. The experiment also sets the basis for a test of LG inequality probing the positional degree of freedom over macroscopic distances. The interaction-free detection method of the atom's position can be adapted well to other systems like matter wave interferometers with large spatial splitting [56-58]. The tendimensional Hilbert space (5 lattice sites with 2 internal states each) of this LG test constitutes a significant advance beyond the simple two-level system, which has been investigated so far. Moreover, the multidimensionality of the Hilbert space [59] can be used in the future to approach the algebraic limit of the correlation function $K$, which is equal to 3. Finally, we should remark on the illustrative value of this violation of the LG inequality, which puts the particle's trajectories in position space at center stage.

\section{ACKNOWLEDGMENTS}

We are indebted to Jean-Michel Raimond for insightful discussions during his stay in Bonn and for critical reading of the manuscript. We thank Ricardo Gomez for his contribution to the experimental apparatus. We acknowledge financial support from NRWNachwuchsforschergruppe "Quantenkontrolle auf der Nanoskala," ERC grant DQSIM, and EU project SIQS. In addition, A. A. acknowledges support from the Alexander von Humboldt Foundation, and C. R. from the BCGS program and Studienstiftung des deutschen Volkes.

\section{APPENDIX A: EXPERIMENTAL APPARATUS}

Each experimental sequence starts with, on average, 1.2 atoms sitting at sufficiently separated lattice sites. Atoms are cooled to the longitudinal ground state first using molasses cooling and then microwave sideband cooling [47], while they are thermally distributed in the direction transverse to the lattice with a temperature of $\approx 10 \mu \mathrm{K}$. Optical pumping initializes $>99 \%$ of the atoms in the $\uparrow$ state. The duration of coin pulses, which are resonant with the hyperfine splitting of $9.2 \mathrm{GHz}$, determines the value of the coin angle $\theta$, with the fair coin pulse lasting $4.5 \mu \mathrm{s}$ (calibrated using Rabi oscillations). The wavelength $\lambda$ of the optical lattice and the two Zeeman hyperfine states are chosen such that the $\uparrow$ state experiences an optical dipole potential originating only from right-handed circularly polarized photons, while the $\downarrow$ state experiences a potential produced by both left- and right-handed circularly polarized photons with relative weights of $7 / 8$ and $1 / 8$, respectively. The lattice depth of $\approx 80 \mu \mathrm{K}$ precludes tunneling between different sites. To implement the state-dependent shift, the two standing waves are displaced by one site with respect to each other, with a linear ramp lasting $21 \mu \mathrm{s}$, which leaves $>99 \%$ of the atoms in the motional ground state, measured with sideband spectroscopy. During the shift, the potential depth experienced by the $\uparrow$ state remains constant, while the one experienced by the $\downarrow$ state is modulated, with a minimum depth of $3 / 4$ in relative units [47]. At the beginning and the end of each sequence, fluorescence imaging determines the position of individual atoms with a measured reliability of $98 \%$, while $2 \%$ of the atoms are erroneously attributed to the adjacent site.

\section{APPENDIX B: DECOHERENCE ANALYSIS}

A four-step quantum walk lasts around $100 \mu$ s without including the duration of $Q\left(t_{2}\right)$. This time should be compared with the spin relaxation time $\left(T_{1}\right)$ and the spin coherence time $\left(T_{2}\right)$. In our system, we measure $T_{1}=107 \pm 5 \mathrm{~ms}$, which is due to Raman scattering of photons from the optical lattice. The $T_{2}$ time is mainly limited by inhomogeneous dephasing due to magnetic field fluctuations and to both scalar and vectorial differential light shifts. Defining $T_{2}$ as the duration of the Ramsey sequence whose interference contrast is reduced to $50 \%$, we measure $T_{2}=229 \pm 16 \mu \mathrm{s}$. We also fit a density matrix description of decohered quantum walks to the measured position distributions [60]. Using the amount of spin decoherence per step as the only free fit parameter, we obtain that spin coherence decreases after each step by $6 \%$ for the fair coin and by $\lesssim 10 \%$ for the other points in Fig. 3, with the reduced chi-squared being $\lesssim 1$.

\section{APPENDIX C: STATISTICAL ERRORS}

In this work, the confidence intervals of the correlation measurements represent $1 \sigma$ statistical uncertainty, which has been computed by fitting a Gaussian profile to the bootstrapped distribution (i.e., the distribution obtained by resampling with replacement). Independently from bootstrapping, we also computed the statistical uncertainties using Monte Carlo resampling, where the statistical errors of position distributions are estimated with binomial statistics (Clopper-Pearson method). The two estimation methods lead to consistent results. For instance, for the fair coin, we obtain $K=1.435 \pm 0.068$ with Monte Carlo and $K=1.435 \pm 0.074$ with bootstrapping. While Monte Carlo analysis requires invariant statistical properties to be valid, bootstrapping analysis remains valid also in the presence of slow drifts of experimental parameters. The close agreement between the two statistical analyses indicates that each correlation measurement of $K$ (lasting about $120 \mathrm{~min}$ ) is performed under constant experimental conditions.

\section{APPENDIX D: SYSTEMATIC ERRORS}

Systematic errors-that is, deviations from the ideal quantum-walk evolution - do not invalidate the result of a LG test, provided that the experiment is performed under constant experimental conditions and that hypothesis (A2) is not contradicted. Nevertheless, we briefly comment on 
the three main mechanisms that bring about systematic fluctuations: (1) Imperfect initialization prepares $<1 \%$ of the atoms in the wrong internal state. However, to derive the LG inequality, a statistical mixture defining the initial state is perfectly admissible. (2) Imperfect reconstruction of the atom's position can be accounted for in terms of a noisy measurement apparatus. (3) Spontaneous flips of the internal state can be accounted for in terms of an additional stochastic process, which also contributes to determining the system's evolution. We estimate that each of these three mechanisms actually affects the position distribution by $<1 \%$, which is less than the statistical uncertainty.

\section{APPENDIX E: VENALITY}

Knee et al. introduced in Ref. [26] the concept of venality $\zeta$ to quantify how often a nonideal negative measurement, i.e., a measurement that could potentially violate (A2), has been performed. In our experiment, with a relative frequency of $1 \%$ (estimated as the upper limit), motional excitations of the unshifted atoms are produced during the measurement of $Q\left(t_{2}\right)$. In addition, spontaneous flips of the internal state that happen during the 200- $\mu$ s-long $Q\left(t_{2}\right)$ measurement could also invalidate hypothesis (A2). This second process, however, occurs with an even smaller relative frequency of $\approx 0.2 \%$. Hence, we quantify the relative frequency of nonideal negative measurements with $\zeta=1 \%$.

Along the lines of Ref. [26], the correlation function $K$ measured in our experiment can be decomposed as $K=1+(1-\zeta) K_{23}^{\text {ideal }}+\zeta K_{23}^{\text {corrupt }}-K_{13}$, where $K_{23}^{\text {ideal }}$ and $K_{23}^{\text {corrupt }}$ denote the correlation function $\left\langle Q\left(t_{3}\right) Q\left(t_{2}\right)\right\rangle$ that has been measured with an ideal negative measurement $Q\left(t_{2}\right)$ and with a corrupted one, respectively. Taking into account the venality $\zeta$, the Leggett-Garg inequality, which is derived from (A1) and (A2), reads $K \leq 1+\zeta\left(K_{23}^{\text {corrupt }}-K_{13}\right)$. From this result, we obtain a new upper bound for $K \leq 1+2 \zeta=1.02$, which is only slightly displaced from the ideal case of 1 .

\section{APPENDIX F: QUANTUM MECHANICAL PREDICTION}

A quantum mechanical calculation shows that, among the possible designations of $Q\left(t_{i}\right)$, the maximal violation of LG inequality is obtained by associating the measurements' results with the extremal values in the permitted range, that is, either +1 or -1 . Other designations, e.g., $Q\left(t_{3}\right)=x / 2$, would lead to a smaller upper bound for $K$.

With our prescription of $Q\left(t_{i}\right)$, we find for a four-step quantum walk, an analytic expression of $K$ as a function of the coin angle $\theta$,

$$
K=\frac{1}{16}[19-4 \cos (2 \theta)+\cos (4 \theta)],
$$

which is the curve plotted as the upper line in Fig. 4. Alternatively, with a dichotomic assignment of $Q\left(t_{2}\right)$ equal to -1 for $(\uparrow, x=-1)$ and to +1 for $(\downarrow, x=+1)$, we obtain

$K=\frac{1}{32}[33-4 \cos (\theta)-4 \cos (2 \theta)+4 \cos (3 \theta)+3 \cos (4 \theta)]$,

which reaches the maximum value of approximately 1.31 , in contrast to 1.5 corresponding to Eq. (F1).

\section{APPENDIX G: QUANTUM WITNESS}

The assignment $Q\left(t_{2}\right)=1$, together with $Q\left(t_{1}\right)=1$ by preparation, implies that the LG inequality (1) can be written in general terms as

$$
K-1=\left(\sum_{x= \pm 1} P\left(t_{2} ; x\right)\left\langle Q\left(t_{3}\right)\right\rangle_{x}\right)-\left\langle Q\left(t_{3}\right)\right\rangle \leq 0 .
$$

This inequality is but one of a family of inequalities, and $K^{\prime}=\left\langle Q\left(t_{2}\right) Q\left(t_{1}\right)\right\rangle-\left\langle Q\left(t_{3}\right) Q\left(t_{2}\right)\right\rangle+\left\langle Q\left(t_{3}\right) Q\left(t_{1}\right)\right\rangle \leq 1$ defines a similar, though independent, inequality built from the same correlation terms [43]. With the choice of $Q\left(t_{i}\right)$ discussed here, we find that $K^{\prime}-1=-(K-1)$. Taken together, these two inequalities imply that $W=|K-1|=0$. The comparison with Ref. [50] allows us to identify $W$ as the first quantum witness in that work.

\section{APPENDIX H: MACROSCOPICITY MEASURE}

Nimmrichter et al. [32] have suggested a universal, objective measure $\mu$ that quantifies the amount of macroscopicity of a mechanical superposition state. In the proposed model, $\mu$ sets a lower limit for the time (expressed in logarithmic scale) during which an electron-chosen as the reference particle-behaves like a "wave" delocalized over distances larger than a certain critical classicalization length scale $\ell$, which represents a phenomenological parameter. The length scale $\ell$ is defined in the model such that quantum superpositions of paths separated by less than $\ell$ preserve their coherence. We estimate for our experiment $\mu=\log _{10}\left(T M_{\mathrm{Cs}}^{2} / m_{\mathrm{e}}^{2}\right) \approx 6.8$ for values of $\ell$ shorter than the maximal separation, $2 \mu \mathrm{m}$, reached during the four-step quantum walk. Here, $M_{\mathrm{Cs}}$ and $m_{\mathrm{e}}$ denote the masses of the Cs atom and of an electron, respectively, and $T$ represents the overall duration of the quantum walk. For values of $\ell$ larger than $2 \mu \mathrm{m}$, the measure $\mu$ as a function of $\ell$ itself behaves, up to an additive constant, as $-2 \log _{10}(\ell / 2 \mu \mathrm{m})$ [32].

[1] V. Giovannetti, S. Lloyd, and L. Maccone, QuantumEnhanced Measurements: Beating the Standard Quantum Limit, Science 306, 1330 (2004). 
[2] J. L. O'Brien, A. Furusawa, and J. Vučković, Photonic Quantum Technologies, Nat. Photonics 3, 687 (2009).

[3] T. D. Ladd, F. Jelezko, R. Laflamme, Y. Nakamura, C. Monroe, and J. L. O'Brien, Quantum Computers, Nature (London) 464, 45 (2010).

[4] E. Schrödinger, Die Gegenwärtige Situation in der Quantenmechanik, Naturwissenschaften 23, 807 (1935).

[5] M. Born, Statistical Interpretation of Quantum Mechanics, Science 122, 675 (1955).

[6] E. P. Wigner, Remarks on the Mind-Body Question, in The Scientist Speculates, edited by I. J. Good (Heinemann, London, 1962).

[7] H. Everett, Many-Worlds Interpretation of Quantum Mechanics, edited by B.S. DeWitt and N. Graham, Princeton Series in Physics (Princeton University Press, Princeton, 1973).

[8] Quantum Theory and Measurement, edited by J. A. Wheeler and W. H. Zurek, Princeton Series in Physics (Princeton University Press, Princeton, 1983).

[9] A. Bassi and G. Ghirardi, A General Argument against the Universal Validity of the Superposition Principle, Phys. Lett. A 275, 373 (2000).

[10] W. H. Zurek, Decoherence and the Transition from Quantum to Classical-Revisited, Los Alamos Sci. 27, 86 (2002).

[11] A. J. Leggett, The Quantum Measurement Problem, Science 307, 871 (2005).

[12] S. L. Adler and A. Bassi, Is Quantum Theory Exact?, Science 325, 275 (2009).

[13] N. D. Mermin, Commentary Quantum Mechanics: Fixing the Shifty Split, Phys. Today 65, 8 (2012); see also the ensuing debate in the December Issue.

[14] M. F. Pusey, J. Barrett, and T. Rudolph, On the Reality of the Quantum State, Nat. Phys. 8, 475 (2012).

[15] A. J. Leggett, Realism and the Physical World, Rep. Prog. Phys. 71, 022001 (2008).

[16] A. Bassi, K. Lochan, S. Satin, T. P. Singh, and H. Ulbricht, Models of Wave-Function Collapse, Underlying Theories, and Experimental Tests, Rev. Mod. Phys. 85, 471 (2013); the many-worlds interpretation provides a third point of view, which, however, appears to be difficult to either verify or falsify.

[17] A. J. Leggett and A. Garg, Quantum Mechanics versus Macroscopic Realism: Is the Flux There When Nobody Looks?, Phys. Rev. Lett. 54, 857 (1985).

[18] A. Palacios-Laloy, F. Mallet, F. Nguyen, P. Bertet, D. Vion, D. Esteve, and A. N. Korotkov, Experimental Violation of a Bell's Inequality in Time with Weak Measurement, Nat. Phys. 6, 442 (2010).

[19] J. P. Groen, D. Ristè, L. Tornberg, J. Cramer, P. C. de Groot, T. Picot, G. Johansson, and L. DiCarlo, PartialMeasurement Backaction and Nonclassical Weak Values in a Superconducting Circuit, Phys. Rev. Lett. 111, 090506 (2013).

[20] M. E. Goggin, M. P. Almeida, M. Barbieri, B. P. Lanyon, J. L. O'Brien, A. G. White, and G. J. Pryde, Violation of the Leggett-Garg Inequality with Weak Measurements of Photons, Proc. Natl. Acad. Sci. U.S.A. 108, 1256 (2011).

[21] J.-S. Xu, C.-F. Li, X.-B. Zou, and G.-C. Guo, Experimental Violation of the Leggett-Garg Inequality under Decoherence, Sci. Rep. 1, 101 (2011).
[22] J. Dressel, C. J. Broadbent, J. C. Howell, and A. N. Jordan, Experimental Violation of Two-Party Leggett-Garg Inequalities with Semiweak Measurements, Phys. Rev. Lett. 106, 040402 (2011).

[23] Y. Suzuki, M. Iinuma, and H. F. Hofmann, Violation of Leggett-Garg Inequalities in Quantum Measurements with Variable Resolution and Back-action, New J. Phys. 14, 103022 (2012).

[24] G. Waldherr, P. Neumann, S. F. Huelga, F. Jelezko, and J. Wrachtrup, Violation of a Temporal Bell Inequality for Single Spins in a Diamond Defect Center, Phys. Rev. Lett. 107, 090401 (2011).

[25] V. Athalye, S. S. Roy, and T. S. Mahesh, Investigation of the Leggett-Garg Inequality for Precessing Nuclear Spins, Phys. Rev. Lett. 107, 130402 (2011).

[26] G. C. Knee, S. Simmons, E. M. Gauger, J. J. L. Morton, H. Riemann, N. V. Abrosimov, P. Becker, H.-J. Pohl, K. M. Itoh, M. L. W. Thewalt, G. A. D. Briggs, and S.C. Benjamin, Violation of a Leggett-Garg Inequality with Ideal Non-invasive Measurements, Nat. Commun. 3, 606 (2012).

[27] G. C. Ghirardi, A. Rimini, and T. Weber, Unified Dynamics for Microscopic and Macroscopic Systems, Phys. Rev. D 34, 470 (1986).

[28] P. Pearle, Combining Stochastic Dynamical State-Vector Reduction with Spontaneous Localization, Phys. Rev. A 39, 2277 (1989).

[29] R. Penrose, On Gravity's Role in Quantum State Reduction, Gen. Relativ. Gravit. 28, 581 (1996).

[30] M. M. Wilde and A. Mizel, Addressing the Clumsiness Loophole in a Leggett-Garg Test of Macrorealism, Found. Phys. 42, 256 (2012).

[31] A. J. Leggett, Testing the Limits of Quantum Mechanics: Motivation, State of Play, Prospects, J. Phys. Condens. Matter 14, R415 (2002).

[32] S. Nimmrichter and K. Hornberger, Macroscopicity of Mechanical Quantum Superposition States, Phys. Rev. Lett. 110, 160403 (2013).

[33] M. Arndt and K. Hornberger, Testing the Limits of Quantum Mechanical Superpositions, Nat. Phys. 10, 271 (2014).

[34] N. Lambert, C. Emary, Y.-N. Chen, and F. Nori, Distinguishing Quantum and Classical Transport through Nanostructures, Phys. Rev. Lett. 105, 176801 (2010).

[35] R. P. Feynman and A. R. Hibbs, Quantum Mechanics and Path Integrals (McGraw-Hill, New York, 1965), problem 2.6.

[36] O. Mandel, M. Greiner, A. Widera, T. Rom, T. W. Hänsch, and I. Bloch, Coherent Transport of Neutral Atoms in Spin-Dependent Optical Lattice Potentials, Phys. Rev. Lett. 91, 010407 (2003).

[37] M. Karski, L. Förster, J.-M. Choi, A. Steffen, W. Alt, D. Meschede, and A. Widera, Quantum Walk in Position Space with Single Optically Trapped Atoms, Science 325, 174 (2009).

[38] H. Schmitz, R. Matjeschk, C. Schneider, J. Glueckert, M. Enderlein, T. Huber, and T. Schätz, Quantum Walk of a Trapped Ion in Phase Space, Phys. Rev. Lett. 103, 090504 (2009).

[39] F. Zähringer, G. Kirchmair, R. Gerritsma, E. Solano, R. Blatt, and C. F. Roos, Realization of a Quantum Walk with 
One and Two Trapped Ions, Phys. Rev. Lett. 104, 100503 (2010).

[40] M. A. Broome, A. Fedrizzi, B. P. Lanyon, I. Kassal, A. Aspuru-Guzik, and A. G. White, Discrete Single-Photon Quantum Walks with Tunable Decoherence, Phys. Rev. Lett. 104, 153602 (2010).

[41] A. Schreiber, K. N. Cassemiro, V. Potoček, A. Gábris, P. J. Mosley, E. Andersson, I. Jex, and C. Silberhorn, Photons Walking the Line: A Quantum Walk with Adjustable Coin Operations, Phys. Rev. Lett. 104, 050502 (2010).

[42] L. Sansoni, F. Sciarrino, G. Vallone, P. Mataloni, A. Crespi, R. Ramponi, and R. Osellame, Two-Particle BosonicFermionic Quantum Walk via Integrated Photonics, Phys. Rev. Lett. 108, 010502 (2012).

[43] C. Emary, N. Lambert, and F. Nori, Leggett-Garg Inequalities, Rep. Prog. Phys. 77, 016001 (2014).

[44] J. von Neumann, Mathematical Foundations of Quantum Mechanics (Princeton University Press, Princeton, 1955).

[45] G. Luiders, Concerning the State-Change Due to the Measurement Process, Ann. Phys. (Amsterdam) 15, 663 (2006).

[46] M. Karski, L. Förster, J. M. Choi, W. Alt, A. Widera, and D. Meschede, Nearest-Neighbor Detection of Atoms in a $1 D$ Optical Lattice by Fluorescence Imaging, Phys. Rev. Lett. 102, 053001 (2009).

[47] N. Belmechri, L. Förster, W. Alt, A. Widera, D. Meschede, and A. Alberti, Microwave Control of Atomic Motional States in a Spin-Dependent Optical Lattice, J. Phys. B 46, 104006 (2013).

[48] P. Kwiat, H. Weinfurter, T. Herzog, A. Zeilinger, and M. A. Kasevich, Interaction-Free Measurement, Phys. Rev. Lett. 74, 4763 (1995).

[49] M. Genske, W. Alt, A. Steffen, A. H. Werner, R. F. Werner, D. Meschede, and A. Alberti, Electric Quantum Walks with Individual Atoms, Phys. Rev. Lett. 110, 190601 (2013).
[50] C.-M. Li, N. Lambert, Y.-N. Chen, G.-Y. Chen, and F. Nori, Witnessing Quantum Coherence: From Solid-State to Biological Systems, Sci. Rep. 2, 885 (2012).

[51] D. Dürr and S. Teufel, Bohmian Mechanics: The Physics and Mathematics of Quantum Theory (Springer, Berlin, 2009).

[52] J. I. Korsbakken, F. K. Wilhelm, and K. B. Whaley, The Size of Macroscopic Superposition States in Flux Qubits, Europhys. Lett. 89, 30003 (2010).

[53] Z. Merali, Quantum Mechanics Braces for the Ultimate Test, Science 331, 1380 (2011).

[54] T. Scheidl, R. Ursin, J. Kofler, S. Ramelow, X.-S. Ma, T. Herbst, L. Ratschbacher, A. Fedrizzi, N. K. Langford, T. Jennewein, and A. Zeilinger, Violation of Local Realism with Freedom of Choice, Proc. Natl. Acad. Sci. U.S.A. 107, 19708 (2010).

[55] J. Gallicchio, A. S. Friedman, and D. I. Kaiser, Testing Bell's Inequality with Cosmic Photons: Closing the SettingIndependence Loophole, Phys. Rev. Lett. 112, 110405 (2014).

[56] A. Alberti, V. V. Ivanov, G. M. Tino, and G. Ferrari, Engineering the Quantum Transport of Atomic Wavefunctions over Macroscopic Distances, Nat. Phys. 5, 547 (2009).

[57] H. Müntinga et al., Interferometry with Bose-Einstein Condensates in Microgravity, Phys. Rev. Lett. 110, 093602 (2013).

[58] S. M. Dickerson, J. M. Hogan, A. Sugarbaker, D. M. S. Johnson, and M. A. Kasevich, Multiaxis Inertial Sensing with Long-Time Point Source Atom Interferometry, Phys. Rev. Lett. 111, 083001 (2013).

[59] C. Budroni and C. Emary, Temporal Quantum Correlations and Leggett-Garg Inequalities in Multilevel Systems, Phys. Rev. Lett. 113, 050401 (2014).

[60] A. Alberti, W. Alt, R. Werner, and D. Meschede, Decoherence Models for Discrete-Time Quantum Walks and Their Application to Neutral Atom Experiments, arXiv:1409.6145 [New J. Phys. (to be published)]. 